\title{
Packet-based Approaches to ATM Cell Policing, and their Effects on Internet Traffic
}

\author{
C. Song
}

Advisory Engineer, $M C I$

2100 Reston Parkway, Reston, Virginia, USA

+1 703.715.7114, +1 703.715.7066 (fax), csong@mci.net

\section{R. Wilder}

Senior Manager, $M C I$

2100 Reston Parkway, Reston, Virginia, USA,

+1703.715.7114, +1 703.715.7066(fax),wilder@mci.net

\section{T. Dwight}

Advisory Engineer, $M C I$

901 International Parkway, Richardson, Texas, USA

+1972.498.1484,+1972.498.1300 (fax),Tim.Dwight@mci.com

\begin{abstract}
IP traffic is commonly carried over ATM networks, using a Variable Bit Rate service. In this configuration, the ATM network typically measures the user's conformance to the subscribed traffic contract, using Usage Parameter Control (also known as policing) at the ATM network ingress. Traditional policing algorithms, which measure the user's rate of transmission at the ATM Layer, can lead to excessive loss when applied to packet data This paper analyses several alternative policing algorithms, which attempt to minimise packet loss while still enforcing the ATM traffic contract. We observe that while some of the proposed algorithms perform better than others, all of them perform better (in terms of IP layer throughput) than traditional cell-based policing. Our study was conducted using traffic samples from the MCI Internet backbone. For this reason, our results may be more credible than those resulting the use of simulation or mathematical models.
\end{abstract}

The original version of this chapter was revised: The copyright line was incorrect. This has been corrected. The Erratum to this chapter is available at DOI: 10.1007/978-0-387-35388-3_42 


\section{INTRODUCTION}

Conventional ATM policing implementations are based on the Generic Cell Rate Algorithm (GCRA) defined in ITU-T Recommendation I.371 (I.371, 1995). To verify conformance with the traffic parameters associated with a given connection, they measure the interarrival times at the ATM layer (i.e., the gaps between the cells). In order to accommodate some measure of variability in this measured rate, they implement what is commonly termed a "leaky bucket". The leaky bucket "drains" at the subscribed rate (PCR or SCR), and "fills" each time an inter-cell gap is judged to be too small. Cells are deemed to be nonconforming, and subjected to some form of punishment (generally either discard or tagging) if the bucket overflows.

Conformance checking as performed by the packet based policing mechanisms discussed in this paper, is based on both the leaky bucket fill, and on where in the encompassing Protocol Data Unit (PDU) the cells lie. In packet based policing, conformance checking is affected by and affects other cells in the same PDU.

The motivation for packet based policing is the fact that dropping one cell implies a loss of the entire packet to which the cell belongs (since the destination host will not be able to reassemble it). To maximise goodput for the higher layer protocol traffic, a packet based policer attempts to minimise the number of packets being policed, i.e., tagged or dropped, while still maintaining network performance and QoS commitments expressed in terms of cells.

The Usage Parameter Control definitions in (I.371, 1995) and (UNI 3.1, 1994) explicitly allow such behaviour. They require only that the number of cells judged nonconforming be within a certain tolerance of the number that would have been so judged by a "perfect policer".

The packet based policing algorithms discussed in this paper mark as nonconforming, all cells subsequent to a nonconforming cell in the same packet. In standard (cell-based) policing, some of the latter cells will typically be judged conforming. Thus packet based policing reduces the number of nonconforming packets (packets in which one or more cell was judged nonconforming) by making room in the leaky bucket for subsequent cells belonging to new packets.

In this paper we analyse the effects of alternative policing algorithms, using traffic traces collected from the MCI Internet backbone, one of the largest, fastest, and busiest U.S. core IP backbones in existence. In the MCI Internet backbone, as in many carrier backbones, customer traffic is aggregated via a series of successively higher speed devices, into a set of core IP routers. The core routers are in turn interconnected via ATM PVCs, through an inner ring of ATM switches. The traces used in this study represent traffic on these PVCs.

Our analysis consisted of feeding this trace data to programs emulating the various policing algorithms, to observe how the associated flow would have fared, had it been subject to the associated policing mechanism. Our main contribution in this study is a set of quantitative results based on actual traffic measured in a production environment typical of IP over ATM in large networks today. 


\section{Applicability}

This study is applicable to IP-over-ATM networks which utilize policing. We note two significant cases in which this configuration is found:

- When the IP and ATM components belong to different administrations, policing will typically be applied by the ATM operator based on subscription agreements between the two. For example, the ATM network may be a public network, and the IP network may belong to an Internet Service Provider (ISP). In the U.S., such an arrangement is quite common.

- When the IP network wishes to offer differentiated service, for example by mapping the IETF's RSVP signaling to ATM signaling and thereby mapping IP flows to ATM connections, policing will typically be used to ensure that the QoS requested at the IP layer, can be guaranteed by the ATM layer.

\section{Structure of this paper}

Section 2 describes the evaluated policing algorithms. In Section 3, we describe our data collection environment and tools. In Section 4, we discuss the results of our study. Section 5 summarises our work.

\section{PACKET BASED POLICING ALGORITHMS}

We evaluated five policing algorithms: one for cell policing and four for packet based policing. The cell policing algorithm is based on the GCRA (I.371, 1995), (UNI 3.1, 1994), (TM 4.0, 1997). We classify the four packet based policing algorithms into fixed bucket size and variable bucket size, depending on their mode of restricting the leaky bucket size. One set, termed FBP-like, for Fixed-size Bucket Policing, defines conformance per the standard GCRA, up to the point that a cell is determined to be nonconforming. From that point until the last cell of the current PDU is received, all cells on the associated connection are considered nonconforming. Such algorithms ensure that the leaky bucket size is not violated.

The other algorithm, which we call VBP for Variable-size Bucket Policing, relaxes the bucket size restriction normally enforced by the GCRA. It allows the leaky bucket to fill beyond its configured size, in order to ensure that the same policing outcome is applied to all cells of a given PDU. If the bucket is over-full when the first cell of a new PDU is received, that cell and all subsequent cells of the PDU to which it belongs are judged to be nonconforming. In this algorithm, either all cells of a given PDU are judged conforming, or they are all judged to be nonconforming. Note that the maximum additional bucket size allowed by this algorithm is given by MTUcells - 1, where MTUcells is the Maximum Transfer Unit of the underlying connection, expressed as a number of cells. For a bursty traffic source, the bucket fill can oscillate around the original bucket size, with an overfill no more than MTUcells - 1. 
We chose to evaluate these two types of algorithms for several reasons. First, between them they encompass a wide range of algorithm behaviour. Second, they are similar to the two widely used packet based cell discard mechanisms, Partial Packet Discard (PPD) and Early Packet Discard (EPD). FBP is like PPD in that a negative policing outcome is applied to all remaining cells of the associated PDU. The $F B P$ algorithms result in different policing outcomes being applied to portions of the same PDU; much as PPD results in the discard of partial PDUs.

$V B P$ is like EPD in its application of the same policing outcome to all cells of a given PDU. Our definition of $V B P$ differs from EPD in that it does not have a separate threshold besides the leaky bucket size, to trigger a change in policer state. Effectively, the $V B P$ bucket size is the triggering threshold, and the extended $V B P$ bucket size is the leaky bucket size plus MTUcells-1.

$V B P$ applies the same policing outcome to all cells of a given PDU, including the last cell. For the $F B P$ algorithms, it is desirable to ensure delivery of the last cell in a PDU regardless of the policing outcome applied to the rest of the PDU, in order to retain the packet boundary indication. If the last cell in a PDU is not delivered, frequently 2 PDUs will fail to be reassembled successfully. This happens because the SAR process unwittingly attempts to reassemble cells of two PDUs into a single PDU. The resulting PDU will almost certainly fail either the length check or the CRC validation performed by the SAR layer at the receiving end system.

We evaluated three variations of $F B P$, which differ in their handling of the last cell in a PDU:

- FBP- $a$ applies the same policing outcome to the last cell, as was applied to the other cells of the PDU

- $\quad F B P-n$ always finds the last cell of a PDU to be conforming

- FBP-g applies to the last cell of the PDU, the result of the standard GCRA policer.

\section{CELL TRACES AND MEASUREMENT ENVIRONMENT}

We obtained cell traces using an internally developed OC3 monitor (Apisdorf, Claffy, Thompson and Wilder, 1996). The monitor is a PC based platform with two Fore Systems ATM interface cards. Each card has a general purpose microprocessor that performs cell timestamping, payload stripping and DMA transfer over the PCI bus to the host memory. One card was used for each direction of an OC3 link.

We collected several trace samples, each consisting of cell arrival records corresponding to several million cells arriving over the same OC3 link. Each record contained the header from one received cell, and a timestamp representing the time at which the last bit of the cell was received. Timestamps were estimated using a $25 \mathrm{MHz}$ hardware clock on the ATM interface card. 


\section{Measurement Accuracy}

It was not possible to schedule the on-card processor each time a cell arrived, due to the overhead associated with processing interrupts. Instead, cells were read into an input FIFO, which was polled within a tight loop such that the per cell processing time was smaller than the OC3 cell inter-arrival time. The processor left this loop when the FIFO was empty.

Loop re-entrance delay plus the per-cell processing delay caused small inaccuracies in the timestamps assigned to the cells. To ensure the accuracy of our study, we analysed the worst cast deviation of this timestamp from the actual arrival time. Our traces show a minimum of 62 clock ticks' difference between the timestamps assigned to consecutive cells. The OC3 cell interarrival time is 2.87 $\mu \mathrm{s}$, or 71.9 ticks of a $25 \mathrm{MHz}$ clock. Cells whose recorded interarrival times were smaller than this theoretical minimum, represent cases where the processor found multiple cells in the FIFO (62 ticks represents the processing time of a cell within the tight loop).

The longest sequence of cells with $62 \mathrm{~ms}$ interarrival times, in any of the recorded traces, was seven (7). Therefore the maximum error between the actual arrival time and the recorded arrival time, was as indicated in equation (1) below.

$7 \times(71.9-62)=69.3$ ticks

Note that this is less than one OC3 cell time (71.9 ticks). We conclude that the error introduced via our method of assigning timestamps, does not significantly impact the validity of our results.

\section{Network Configuration}

We collected traces at the Washington DC POP on MCI's Internet backbone. We inserted the OC3 monitor into the OC3 link between an ATM switch and an IP router at this POP. The switch is connected to other switches across the country via OC 3 and OC12 links. The router, considered part of the backbone core, forms the boundary between the ATM network and the IP access to the backbone. Connected to this core router over various media are many access routers that provide hundreds of customer connection ports. Ranging from DS0 to DS3 in speed, these ports connect MCI's customers including NAPs, regional networks, and corporate leased lines.

ATM cells traced by the OC3 monitor originate and terminate at core routers' ATM ports. Each VC seen by the OC3 monitor is backbone edge to edge, aggregating IP traffic to/from a large number of customers behind the access routers. In order to achieve maximum statistical multiplexing, the ATM interfaces on the core routers do not implement traffic shaping (i.e., they make no attempt to control their rate of transmission); nor do the ATM switches implement policing.

This study analyses what would have been the result, had the VCs in question been subjected to each of the proposed policing implementations. The traffic 
parameters (SCR and MBS) of the VCs were varied over a range of possible values, and for each set of values, the traces were subjected to a simulation of each policing mechanism. Policing outcomes were collected and analysed, as discussed in the next section.

\section{EVALUATION RESULTS}

We used three criteria to evaluate the packet based policing algorithms:

- The number of packets containing one or more cells judged to be nonconforming (for simplicity, referred to as nonconforming packets). To ensure high packet-level goodput, it is desirable to minimise this number.

- The ratio of nonconforming cells to nonconforming packets. It is desirable to maximise this number.

- Number of multi-cell packets in which the last cell was judged nonconforming but one or more of the previous cells was judged conforming. It is desirable to minimise this number, as such "partially conforming packets" can lead to a blurring of packet boundaries, and thus to the loss of multiple packets due to reassembly failure.

All the trace data exhibit essentially the same behaviour for all the evaluated algorithms. In the interest of brevity, we focus our presentation of results on one data trace, collected on November 61996 at about 13:00, containing 10618902 cells and 1484268 packets. 


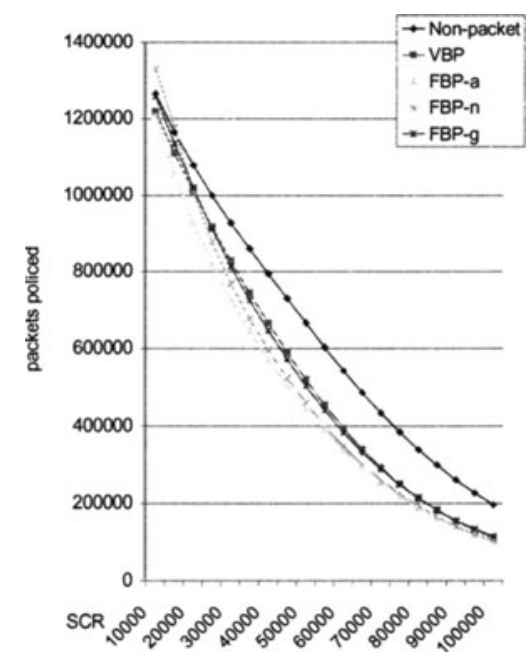

Figure 1 - packets policed $(\mathrm{MBS}=50)$

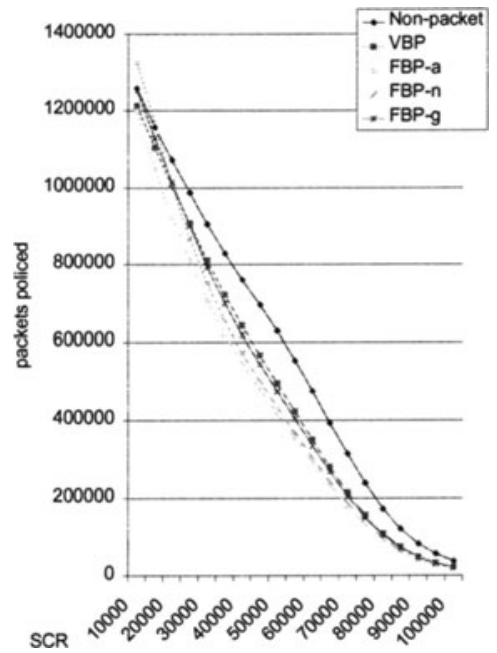

Figure 2 - packets policed $(\mathrm{MBS}=200)$

Figures 1 and 2 show the number of packets judged nonconforming, by each of the evaluated algorithms*. Figure 1 is for Maximum Burst Size (MBS) set to 50 cells, and Figure 2 is for MBS set to 200 cells. The $\mathrm{Y}$ axis indicates the number of "policed packets". The X axis indicates the Sustainable Cell Rate (SCR) setting, ranging from 10000 to 100000 cells/second with an increment of 5 000. The Peak Cell Rate (PCR) is in all cases assumed to be set to the OC3 line rate.

As expected, the cell-based UPC algorithm produces the highest number of policed packets. The $F B P-a$ produces the least number of policed packets. All FBP algorithms produce lower numbers of policed packets than $V B P$ does, except when the SCR is very low. Figures 3 and 4 suggest a possible explanation, showing the number of policed packets vs. the packet size (expressed as the number of cells in a packet). Both figures have MBS 200. Figure 3 is for the SCR setting of 10000 and Figure 4 is the same for $\mathrm{SCR}=25000$. The graphs indicate that those algorithms showing fewer policed packets tend to show more policed packets of size 5 cells or greater, and far fewer policed packets of size less than 5 cells. When combined, the total numbers of policed packets are lower than those produced by algorithms that tend to police fewer large packets and more small packets.

\footnotetext{
"The figures use the phrase "policed packet" to refer to a packet in which one or more cells was judged to be non-conforming.
} 


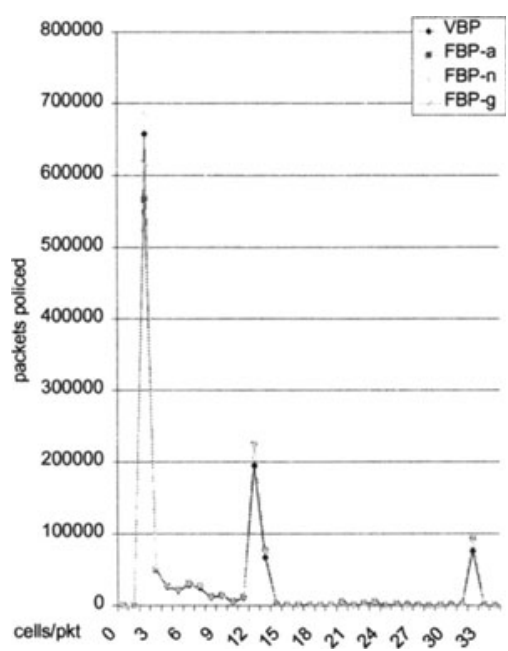

Figure 3 - policed packets vs. packet size $(\mathrm{MBS}=200, \mathrm{SCR}=10000)$

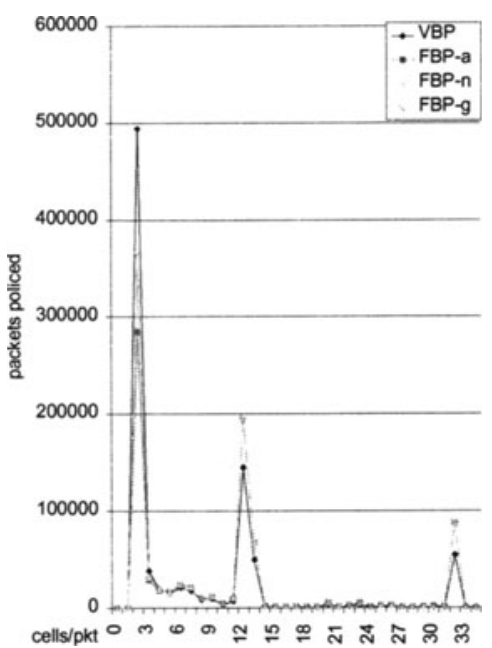

Figure 4 - policed packets vs. packet size $(\mathrm{MBS}=200, \mathrm{SCR}=25000)$

We observe that the effects of packet based policing algorithms appear related to the IP packet burst distribution and size characteristics. Large packets will incur large emission delay, causing other packets to queue up behind them (so-called "ACK compression", is an example of this phenomenon). Since most IP packets are small, such queued packets tend to be small. The result is a "packet train" consisting of a large packet followed by several smaller packets, which arrive back-to-back at the next multiplexing point in the network.

When such packet trains arrive, they cause different policing algorithms to behave differently. $V B P$ is likely to find all cells of the large packet conforming, even if to do so it must allow its leaky bucket to surpass its configured size. Once the end of the large packet is detected, it may be necessary to mark several subsequent packets nonconforming in order to allow the leaky bucket to drain.

With the FBP algorithms, the rigidly enforced bucket size prevents the bucket from becoming over-full. As a result, fewer subsequent packets are found to be nonconforming.

Figures 3 and 4 show that when SCR is decreased, the number of small packets policed by the $F B P$ algorithms increases. This effect is most pronounced with $F B P-n$. At the SCR setting of 10000 , the $F B P-n$ algorithm policed more packets than $V B P$, perhaps due to $F B P-n$ never judging the last cell of a PDU to be nonconforming. 


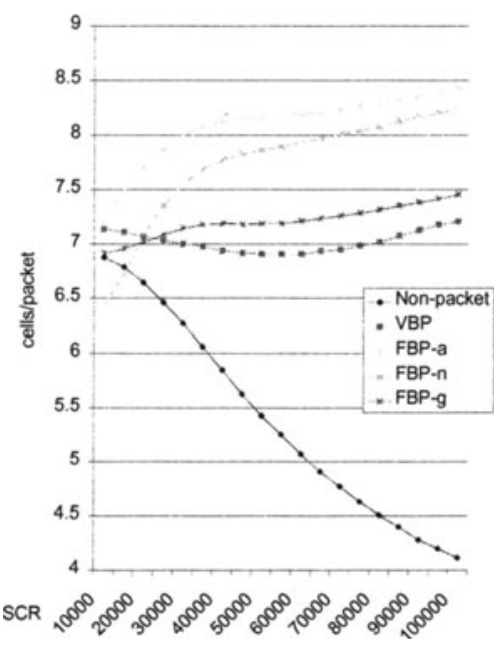

Figure 5 - policed cells per packet $(\mathrm{MBS}=50)$

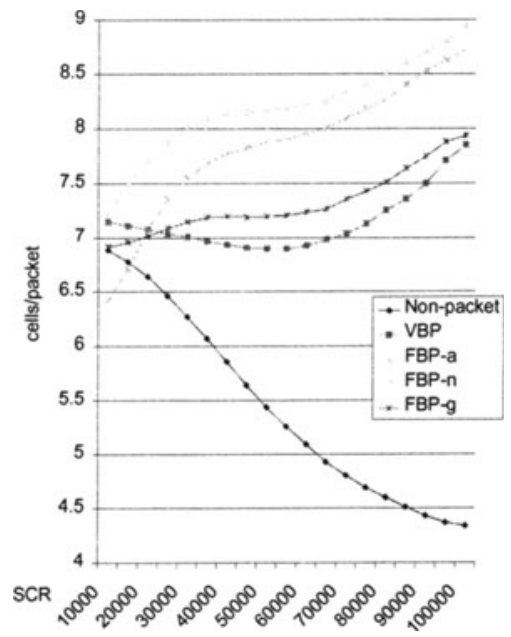

Figure 6 - policed cells per packet $(\mathrm{MBS}=200)$

For our first evaluation criterion (minimisation of the number of policed packets), FBP- $a$ performed the best, and the $V B P$ and cell-based policing performed the worst. For the second criterion (maximisation of the ratio of policed cells to policed packets), FBP- $a$ again performs the best, and $V B P$ and cell-based policing perform the worst.

The third criterion was the algorithm's ability to preserve packet boundaries. This criterion is applicable only to conventional cell-based policing, FBP-a and $F B P$-g. The other two, $V B P$ and $F B P-n$, do not produce partial packets. Figures 7 and 8 show the numbers of partial packets produced with the MBS at 50 and 200 respectively. When the SCR setting is reasonably high, the FBP-a performs the best by producing the lowest number of partial packets. The cell-based policing algorithm produces the highest number of partial packets (i.e., performs the worst) while FBP-g's number lies in between. 


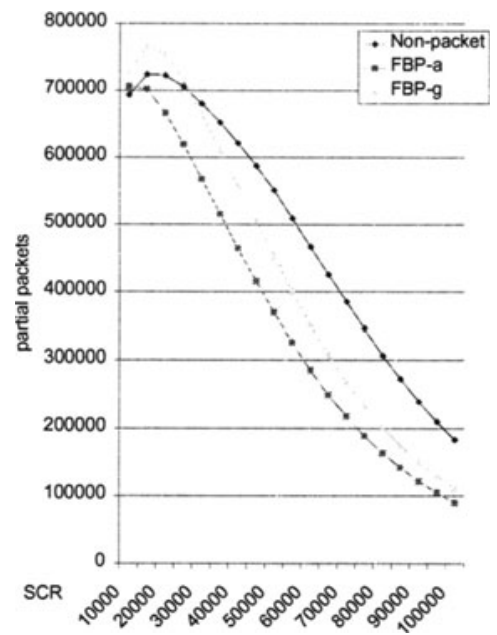

Figure 7 - partial packets $(\mathrm{MBS}=50)$

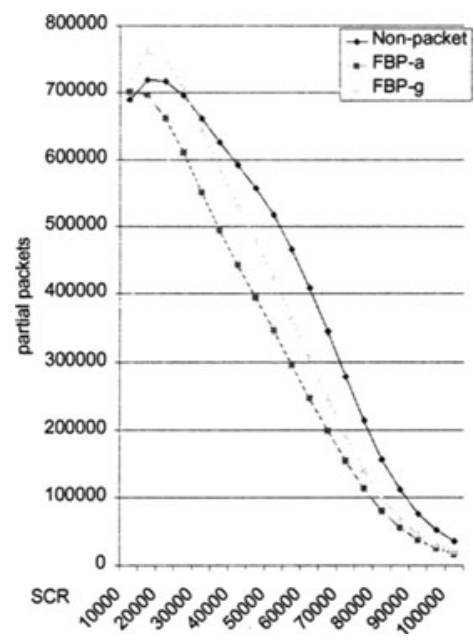

Figure 8 - partial packets $(\mathrm{MBS}=200)$

The cell-based algorithm produces the lowest number when the SCR is set very low. We attempt to explain this by pointing out the effects of two factors: packet size and packet bursts. As we saw earlier, setting the SCR low leads to all packet based policing algorithms having the same number of policed packets of small size, but they still police a higher number of large packets than does the cellbased algorithm. With a low SCR, the effect of packet bursts intensifies such that the last cells of packets that would otherwise be judged as conforming (with a higher SCR), are judged as nonconforming.

The following table summarises the results.

Table 1 - Relative performance of policing algorithms ( 1 = best, 5 = worst $)$

\begin{tabular}{lrrrrr}
\hline & \multicolumn{5}{c}{ Policing Algorithm } \\
& Cell-based & FBP-a & FBP- $n$ & FBP-g & VBP \\
\hline Packets policed & 5 & 1 & 2 & 3 & 4 \\
Ratio of policed cells to policed packets & 5 & 1 & 2 & 3 & 4 \\
Preservation of packet boundaries & 4 & 2 & 1 & 3 & 1 \\
\hline
\end{tabular}




\section{SUMMARY AND FURTHER WORK}

Our analysis of the 5 different policing algorithms using actual measured traffic suggests that packet based policing algorithms provide better service to IP data than does the traditional cell-based policer. Among packet based policing algorithms, the choice is between $F B P-a$ and $F B P-n$. For reasonable settings of MBS and with the SCR set to above 25000 for an OC3 line, $F B P-n$ is a good choice, since it entails the second lowest number of policed packets, only slightly more than with $F B P$ - $a$ 's, but the algorithm preserves packet boundaries while $F B P$ $a$ can not. With a low SCR, FBP- $n$ 's number of policed packets becomes substantially higher and the trade-off between the number of policed packets and preservation of packet boundary is no longer straightforward.

In this work, we ignored the effects of packet sizes on policing effects. We hope to next develop a methodology to investigate this effect, so as to judge packet based policing algorithms as more of weighted sum (e.g., associate a weight with packet type and size) rather than a simple count. We will also examine the synergy between the packet-based policing and the packet based cell discard such as EPD/PPD with and without CLP $=1$ discard. We will need to gain a better understanding of the effects on policing by burst patterns by examining burst characteristics in greater detail.

\section{ACKNOWLEDGMENT}

This material is based on work sponsored by the National Science Foundation, grant NCR-9321047. The very high speed Backbone Network Service (vBNS) project is managed and co-ordinated by MCI communications Corporation under sponsorship of the National Science Foundation. The Government has certain rights to this material. Any opinions, findings, and conclusions or recommendations expressed in this material are those of the authors and do not necessarily reflect the views of the National Science Foundation.

\section{REFERENCES}

ITU-T Study Group 13 (1995), Recommendation I.371 "Traffic Control and Congestion Control in B-ISDN"

The ATM Forum (1994), "ATM User Network Interface Specification, Ver. 3.1"

The ATM Forum (1997), "Traffic Management Specification, Version 4.0"

J. Apisdorf, K. Claffy, K. Thompson and R. Wilder (1996) "OC3MON: Flexible, Affordable, High Performance Statistics Collection”, LISA X, September 1996 


\section{BIOGRAPHIES}

\section{C huck Song}

Chuck Song received his Ph.D. degree in Computer Science from the University of Wisconsin 1989. From 1989 to 1994 he worked in IBM. Chuck was on the IP router development team for the NSFNet project. Since 1995 he has been on the vBNS project engineering team in MCI Internet Engineering. Chuck has worked on the design of Concert Internet Plus as well as the engineering of vBNS.

\section{Rick Wilder}

Rick Wilder is the senior manager of MCI's Internet Technologies group. He was one the original members of the design and implementation team for MCI's commercial internet backbone, and manages the engineering team for the veryhigh-speed Backbone Network Service (vBNS) project sponsored by the National Science Foundation. Rick has an MS degree in Computer Science from the American University.

\section{Tim Dwight}

Tim Dwight is responsible for ATM network architecture and traffic engineering, for MCI's commercial ATM service. He holds an MS in Computer Science from the University of Kansas (1985) and holds several patents related to telecommunications. 